\title{
Evolutionary responses to environmental stress by the pitcher-plant mosquito, Wyeomyia smithii
}

\author{
PETER ARMBRUSTER*, WILLIAM E. BRADSHAW, ANGELA L. STEINER \\ \& CHRISTINA M. HOLZAPFEL \\ Department of Biology, University of Oregon, Eugene, OR 97403-1210, U.S.A.
}

\begin{abstract}
We performed truncation selection for increased fitness $\left(r_{\mathrm{c}}\right)$ under conditions of chronic stress from the combined effects of low nutrients and high temperature, representative of extremes likely to be encountered in nature by the pitcher-plant mosquito, Wyeomyia smithii. We performed selection on geographical parental populations and their hybrids to determine whether hybridization would facilitate or constrain adaptation under our selection protocol. The stressful environment decreased fitness $\left(r_{\mathrm{c}}\right)$ by $54 \%$ averaged across all populations relative to near-optimal conditions. After $\approx 10$ generations of selection under chronically stressful conditions, exactly one-half of the parental and one-half of the hybrid populations had gone extinct. Thus hybridization had no effect on the likelihood of population persistence. Fitness $\left(r_{\mathrm{c}}\right)$ of the surviving populations did not show any response to selection. Despite initial hybrid vigour under stressful conditions, the fitness $\left(r_{\mathrm{c}}\right)$ of surviving hybrid populations was either equal to, or worse than, the fitness $\left(r_{\mathrm{c}}\right)$ of surviving parental populations after $\approx 10$ generations of selection. These results suggest that outcrossing populations to augment genetic variation and facilitate adaptation to a rapidly changing environment may not be useful over longer time scales, even in cases where hybridization does initially increase fitness. Although we detected no direct response to selection for increased fitness $\left(r_{\mathrm{c}}\right)$ under lifetime chronic stress, selected populations showed a strong correlated response for survivorship through transient, acute heat and desiccation shock. In evaluating how organisms might respond to future climate change, biologists must maintain a clear distinction between lifelong performance in chronically stressful environments and short-term survivorship through transient, acute stress.
\end{abstract}

Keywords: environmental stress, extinction, shock tolerance, thermal adaptation, Wyeomyia smithii.

\section{Introduction}

Understanding the role of abiotic stress in influencing the abundance and distribution of organisms has been an historically important theme in the development of ecology and evolutionary biology (Andrewartha \& Birch, 1954; Hoffmann \& Parsons, 1991), and the effects of global climate change are generating renewed interest in this topic (Kareiva et al., 1993). Estimates of climate change predict a $1-4.5^{\circ} \mathrm{C}$ increase in mean global temperature over the next 100 years, with greater changes at extreme latitudes (Kattenberg et al., 1996). In the past, most organisms are thought to have responded to climate

*Correspondence. Present address: Institute of Zoology, Zoological Society of London, Regent's Park, London NW1 4RY. E-mail: peter.armbruster@ioz.ac.uk change by latitudinal or altitudinal migration (Bartlein \& Prentice, 1989). However, because of current high levels of habitat fragmentation caused by anthropogenic disturbance, the ability of many organisms to survive future climate change may depend crucially on evolutionary adaptation (Lynch \& Lande, 1993).

In considering management strategies that might facilitate persistence and adaptation in the face of a rapidly changing climate, intraspecific hybridization is one option that is likely to be proposed. The introduction of migrants from distant, genetically differentiated populations is already being discussed as a tool to augment the genetic variation of inbred and isolated populations (Spielman \& Frankham, 1992), and such strategies might be proposed in the future to facilitate adaptation to conditions of global climate change. However, outbreeding depression has the potential to 
compromise the success of such strategies, and our understanding of the dynamics of outbreeding depression is currently limited (Lynch, 1991).

Most experiments that have been designed to assess adaptive responses to stress have measured levels of heritable variation for shock tolerance rather than the fitness of populations encountering chronic stress over the entire life cycle (Hoffmann \& Parsons, 1991). These studies often make the implicit assumption that tolerance of transient, acute shock is genetically correlated to overall fitness under conditions of chronic, lifetime stress. However, there are currently few studies which examine this critical assumption. Furthermore, it is becoming increasingly apparent that the performance of individual fitness correlates may not accurately reflect more comprehensive, composite indices of fitness (Armbruster et al., 1997).

In this study, we have taken particular care to measure a composite index of fitness spanning the whole life cycle to evaluate hypotheses regarding fitness and adaptation under stressful conditions. We utilized capacity for increase, $r_{\mathrm{c}}$ (Bradshaw \& Holzapfel, 1989; Armbruster et al., 1997), as a measure of fitness under environmental stress. We imposed truncation selection for increased $r_{\mathrm{c}}$ under chronic conditions of high temperature and low nutrient availability for $\approx 10$ generations in replicated geographical parental and hybrid populations of the pitcher-plant mosquito, Wyeomyia smithii (Coquillet). We imposed selection under conditions that were, at the outset, designed to force extinction in approximately half of the populations. By imposing such stringent selection, our aim was to examine processes of extinction and adaptation at the boundary of population persistence. We then address two questions: (i) how have different strategies of population hybridization facilitated or constrained persistence and adaptation in a chronically stressful environment? (ii) has selection for increased performance under chronic stress caused a correlated response in resistance to acute heat and desiccation shock?

\section{Materials and methods}

\section{Collection, fitness assay, and selection}

Adult $W$. smithii lay their eggs within the water-filled leaves of the carnivorous purple pitcher plant, Sarracenia purpurea L., wherein developing larvae utilize decomposing prey provided by the host plant as a food source. The geographical distribution of $W$. smithii follows that of its host plant from the Gulf of Mexico north to Labrador and west to Saskatchewan. For this study, $\approx 2000$ larvae were collected from $\approx 50-200$ pitcher plants at each of four localities (Fig. 1) during early

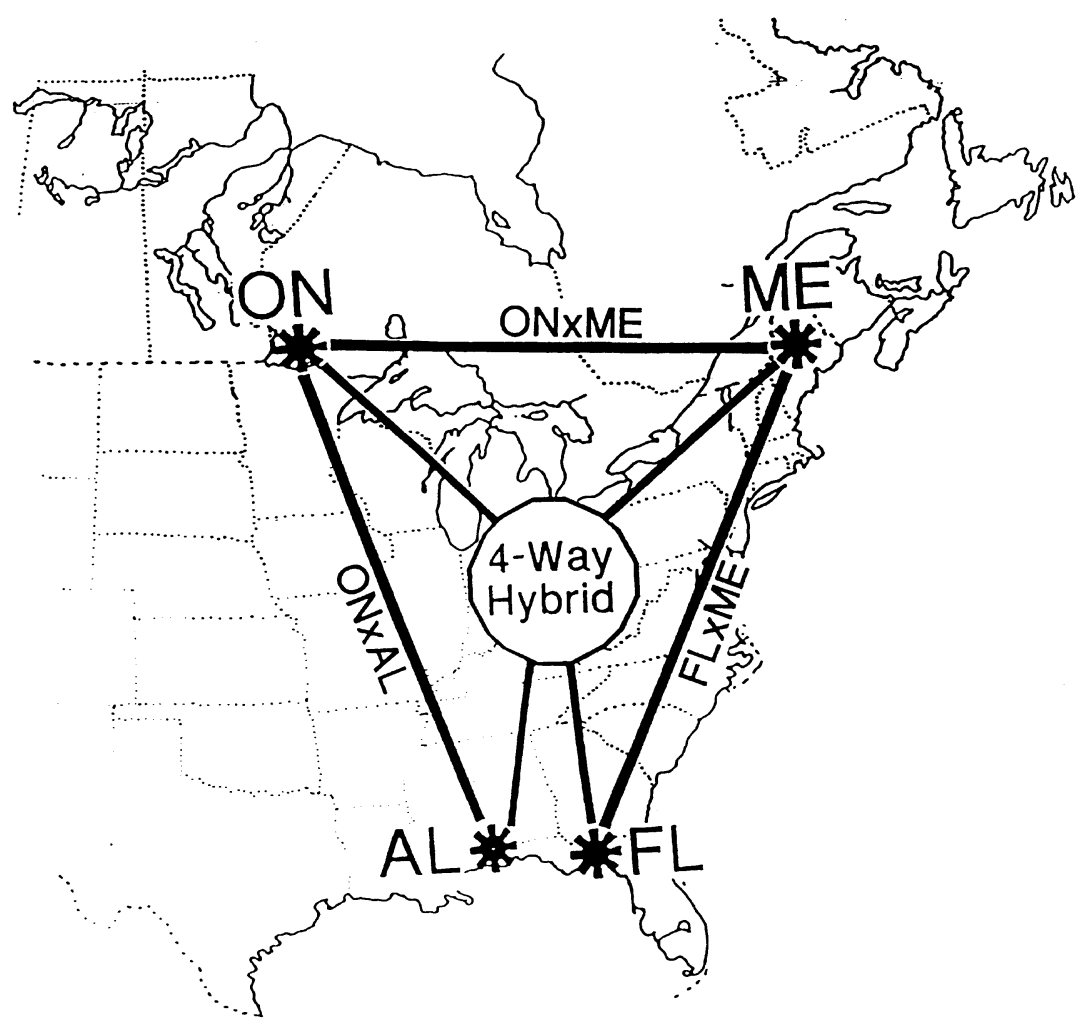

Fig. 1 Geographical origin of parental populations of Wyeomyia smithii in Ontario (ON), Maine (ME), Alabama (AL) and Florida (FL). These populations correspond to DL, $\mathrm{KC}$, LI and WI, respectively, in other studies from this laboratory. Solid lines represent patterns of hybridization used in this experiment. Lines between any two populations indicate crosses performed to generate 2-way hybrid populations. The 4-way hybrid populations were generated by crossing $(\mathrm{ON} \times \mathrm{AL})$ hybrids with $(\mathrm{FL} \times \mathrm{ME})$ hybrids (see Methods).

(C) The Genetical Society of Great Britain, Heredity, 83, 509-519. 
spring when $100 \%$ of the genotypes in the population were available for sampling as overwintering larvae. After collection, larvae from all four populations were run through four generations in the laboratory under near-optimal conditions before the initiation of experiments.

The general outline of this study is presented in Fig. 2. During the initial four generations of laboratory rearing, each generation was composed of between 200 and 500 adult mosquitoes. Thereafter, stock from each of the four localities (parental populations) were used to generate 2-way and 4-way hybrid populations (Fig. 1).

First, we generated hybrid and reciprocal hybrid populations between southern and northern populations $(\mathrm{ON} \times \mathrm{AL}, \mathrm{FL} \times \mathrm{ME}$; Fig. 1$)$ while maintaining the parental stocks. Parental stocks in the $F_{4}$ laboratory generation were split into halves: one half was mass swarmed to regenerate the parental populations and the other half was outcrossed to generate the $F_{1}$ and reciprocal $F_{1}$ hybrid populations. In the next $\left(F_{5}\right.$ laboratory) generation, parental stocks were again divided and mass swarmed to regenerate the parental populations and outcrossed $(\mathrm{ON} \times \mathrm{AL}, \quad \mathrm{FL} \times \mathrm{ME}$, $\mathrm{ON} \times \mathrm{ME}$; Fig. 1) to generate the $\mathrm{F}_{1}$ and reciprocal $\mathrm{F}_{1}$ 2-way hybrid populations.

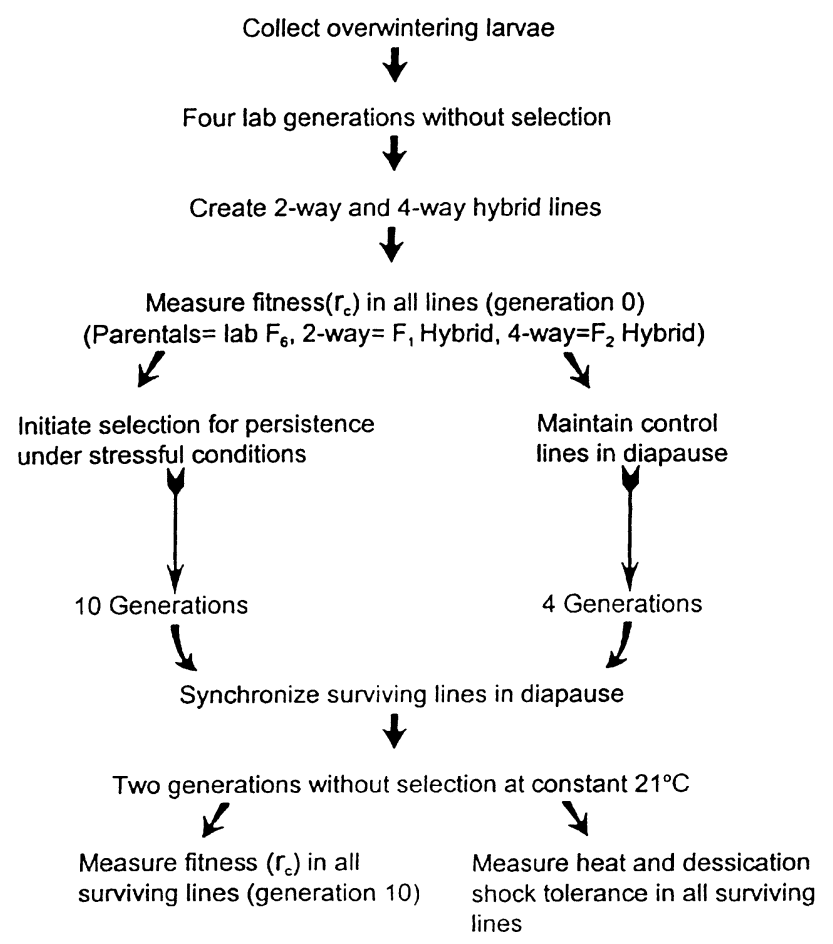

Fig. 2 Outline of experimental design used to examine persistence under, and adaptation to, stressful conditions as well as correlated changes in heat- and desiccation-shock tolerance.
The original $F_{1} s$ and reciprocal $F_{1} s$ between southern and northern populations were crossed to produce four 4-way hybrid populations. These 4-way hybrid populations contained nuclear genes from each of the four parental populations, but each 4-way hybrid population contained a grandmaternal contribution (cytoplasm and mtDNA) from only one of the four parental populations. For example, the 4-way hybrid population with an Ontario grandmaternal contribution was constructed by mating $\mathrm{F}_{1} \mathrm{ON} \odot \times \mathrm{AL}_{\delta} \hat{\jmath}$ females with $\mathrm{F}_{1} \mathrm{FL}_{+} \times \mathrm{ME}_{\widehat{\delta}}$ males. In maintaining the parental populations and generating the hybrid populations, between 96 and 187 adults of each sex were used. These procedures yielded 14 populations in the laboratory $F_{6}$ generation: four parents, six 2-way hybrids, and four 4-way hybrids.

All experiments to evaluate fitness $\left(r_{\mathrm{c}}\right)$ were run with replicate uniform-aged cohorts, where each cohort consisted of 40 larvae placed on the day of hatching into $30 \mathrm{~mL}$ of distilled water in a single leaf of $S$. purpurea on an intact plant. The plants were placed in $7.6 \mathrm{~L}$ terraria (two plants per terrarium), which were then placed in one of two controlled-environment rooms. One controlled-environment room was programmed to provide near-optimal conditions, the second to impose thermal and hydric stress. In both rooms, light consisted of a long-day photoperiod ( $\mathrm{L}: \mathrm{D}=18: 6$, including two, $1 / 2$-hour transitory twilights) that promoted continuous (nondiapause) development in all populations (Bradshaw \& Lounibos, 1977). Temperature consisted of a smooth, sine-wave thermoperiod that lagged the photic cycle by $3 \mathrm{~h}$ (Bradshaw, 1980). To mimic the natural prey-capture cycle of leaves (Bradshaw, 1983), developing larvae were fed whole, freeze-dried Drosophila melanogaster in four weekly instalments.

Details of the procedure for measuring $r_{\mathrm{c}}$ have been provided in earlier papers (Bradshaw \& Holzapfel, 1989; Armbruster et al., 1997). Briefly, eggs from each population were collected from large adult cages, and on the day of hatching first instars from parent or hybrid populations were used to establish uniformed-age cohorts consisting of 40 larvae. Five replicate cohorts per population were established. Individual leaves were checked for pupal development and pupae were removed every Monday-Wednesday-Friday to a dish of distilled water in $0.95 \mathrm{~L}$ single-cohort cages provided with pesticide-free raisins for adult nutrition and a freshly cut leaf of $S$. purpurea. Pupal exuviae (indicating successful adult eclosion) were removed from adult cages every Monday-Wednesday-Friday and used to calculate survivorship ( $\%$ adult eclosion). Eggs were collected every Monday-Wednesday-Friday and used to calculate fecundity (total eggs per ecdysed female) and mean generation time, $T=\Sigma x E_{x} / \Sigma E_{x}$ where $E_{x}$ is the 
number of eggs produced by the cohort at age $x$ in days since oviposition of the cohort. The number of larvae hatching from each collection of eggs was recorded, and fertility ( $\%$ hatching) was calculated as the number of larvae hatching/total number of eggs. Then, for each cohort of larvae raised in an individual leaf, we calculated cohort replacement rate as $R_{0}=$ (fertility)(survivorship)(fecundity) and capacity for increase as $r_{\mathrm{c}}=\ln \left(R_{0}\right) / T$.

Near-optimal conditions The near-optimal conditions provided a control against which the effects of the stressful environment could be assessed. Near-optimal conditions consisted of a warm: $\mathrm{cool}=29: 13^{\circ} \mathrm{C}($ mean $=$ $21^{\circ} \mathrm{C}$ ) smooth, sine-wave thermoperiod, a constant $80 \%$ relative humidity, and a diet producing maximum survivorship to adulthood: 50 flies per cohort on the day of hatching and 200, 100 and 50 flies at successive weekly intervals (10 flies per larva).

Stressful conditions The stressful conditions were designed to impose ecologically realistic, broad physiological stress near the threshold of population persistence over the entire life cycle. Stressful conditions consisted of a warm: $\mathrm{cool}=35: 18^{\circ} \mathrm{C} \quad\left(\right.$ mean $\left.=27^{\circ} \mathrm{C}\right)$ smooth, sine-wave thermoperiod with a high: low $=$ $80: 60 \%$ smooth, sine-wave relative humidity cycle where the daily low humidity occurred at peak temperature. Larvae were provided a reduced diet of 25 flies on the day of hatching and 100, 50 and 25 flies at successive weekly intervals (five flies per larva).

We determined fitness $\left(r_{\mathrm{c}}\right)$ under both near-optimal and stressful conditions in each of the 14 populations before selection ( $F_{6}$ laboratory generation). We then used the same base populations to establish control populations and initiate selection. The control populations were maintained as diapausing larvae on an unambiguous short day ( $\mathrm{L}: \mathrm{D}=8: 16)$ at $21 \pm 0.5^{\circ} \mathrm{C}$. Every 4 months they were reared to adulthood under near-optimal conditions and their resulting offspring were again placed on short days at $21^{\circ} \mathrm{C}$ to induce and maintain diapause. Each generation was composed of at least 100 male and 100 female adult mosquitoes, and these populations were run through a total of four generations during the course of the selection experiment. This protocol was used to increase the generation length of the control populations, therefore minimizing the opportunity for adaptation to the laboratory environment.

To initiate the selected populations, replicate cohorts were established in individual leaves under stressful conditions as described above. Four leaves were established on the first week, and three leaves were established during the second and third weeks. Thus, each population was established with a census population size of 400 larvae. After the third week, one leaf was set up on Tuesday and Friday of each week. At the beginning of week three, leaves were checked every Tuesday and Friday for pupae, and the first five male and five female pupae from a leaf were transferred to a populationspecific adult mass-swarm cage maintained under stressful conditions.

By selecting the first five males and five females to emerge from a leaf, we simultaneously selected fastdeveloping, fecund, and long-lived individuals. Development time (number of days from hatching to pupation) and pupal mass are negatively phenotypically correlated in populations of $W$. smithii (Bradshaw, unpubl. obs.). Furthermore, development time is the primary determinant of mean generation time (Moeur \& Istock, 1980), and pupal mass is the primary determinant of gross lifetime fecundity and adult longevity in W. smithii (Moeur \& Istock, 1980; Bradshaw \& Holzapfel, 1992). Leaves that had not produced at least five male and five female pupae within 50 days were terminated.

Adult cages consisted of 7.6 L plastic buckets with the sides cut out and covered with mesh screening. The bottoms of the cages were lined with $3 \mathrm{~mm}$ thick chromatography paper which was moistened daily. All cages were provided with pesticide-free raisins. A freshly cut $S$. purpurea leaf was added to the cages from Friday to Wednesday to stimulate oviposition.

Eggs were collected from the adult cages on Monday and Wednesday and any hatched larvae were removed. On Tuesday and Friday, if between 20 and 40 first-instar larvae had hatched, these larvae were used to set up new cohorts and excess larvae were discarded. The number of individual freeze-dried D. melanogaster (food) and water volume for each leaf with fewer than 40 larvae was adjusted to maintain constant per-capita nutrition and density levels in all leaves. Until the end of the 10th week of selection, if an adult cage did not produce at least 20 first instars on the day of leaf set-up, a leaf was set up using first instars generated by the control populations. Subsequent to the 10th week $(\approx 1.6$ generations), if an adult cage did not produce at least 20 first instars on the day of leaf set-up, no leaf was established for that population. Up to two leaves per week, depending on egg production, were set up twice a week (one on Tuesday and one on Friday), for 61 weeks, and pupae collected for an additional seven weeks. Assuming a generation time of 45 days, selection was performed for $\approx 10$ generations. A population was considered extinct when no adults or larvae were alive in that population.

After $\approx 10$ generations of selection, eggs were collected from adult cages every Monday-Wednesday-Friday and transferred to short-day $(\mathrm{L}: \mathrm{D}=8: 16)$ at a constant $21^{\circ} \mathrm{C}$ to synchronize all of the surviving populations in 
diapause. To control for parental and grandparental effects (Crill et al., 1996), control and selected populations for each surviving population were then run through two generations on long day (L:D $=18: 6)$ at constant $21^{\circ} \mathrm{C}$ before fitness $\left(r_{\mathrm{c}}\right)$ was measured for five cohorts from each population under stressful conditions as described above for before selection.

\section{Shock tolerance}

We tested for a correlated response in tolerance to acute transient heat and desiccation stress in populations that persisted under our selection protocol. In the same generation used to assess the response to selection for increased fitness $\left(r_{\mathrm{c}}\right)$ under stress, we assayed larval heatshock tolerance, adult heat-shock tolerance, and adult desiccation resistance in the control and selected populations for six of the seven populations that persisted under the selection protocol. An insufficient number of individuals in the $\mathrm{FL}_{+} \times \mathrm{ME}_{\widehat{\sigma}}$ 2-way hybrid selected population was available to measure shock tolerance in this population.

We chose conditions necessary to cause intermediate levels of mortality in control populations over a short time period. First instars were collected on the day of hatching and maintained in 100-mL Petri dishes under standard conditions of food and density. Larval heatshock tolerance was assessed by subjecting 60 one-to three-day-old fourth instars from each population to $5 \mathrm{~min}$ at $42.5^{\circ} \mathrm{C}$. Four vials with $20 \mathrm{~mL}$ distilled water were placed in a Napco water bath. After allowing the temperature in the vials to equilibrate, 15 larvae were removed from a $21 \pm 1^{\circ} \mathrm{C}$ controlled-environment room and added to each vial. Control and selected populations were paired, and each population was run in two blocks on separate days, with 30 control population larvae and 30 selected population larvae in each block. All blocks were run at $3 \mathrm{PM} \pm 1 \mathrm{~h}$ on subsequent days to control for any circadian component contributing to heat-shock tolerance (Pittendrigh, 1961). Larvae were placed in distilled water at room temperature $\left(22 \pm 1^{\circ} \mathrm{C}\right)$ for two hours subsequent to heat shock, after which survivorship was assessed. Larvae were considered dead if they did not move when placed under a light (larvae are normally negatively phototactic) and gently prodded with a blunt probe.

Adult heat-shock tolerance was measured by placing one- to five-day-old adults maintained at $21 \pm 1{ }^{\circ} \mathrm{C}$ in $2.5 \times 7.5 \mathrm{~cm}(\mathrm{D} \times \mathrm{L})$ cylindrical mesh cages at $54^{\circ} \mathrm{C}$ for $7 \mathrm{~min}$. As above, control and selected populations were paired, and each population was run in two blocks on separate days, with 30 control population adults and 30 selected population adults in each block. All blocks were run at $3 \mathrm{PM} \pm 1 \mathrm{~h}$. Adults were placed at room temperature $\left(22 \pm 2^{\circ} \mathrm{C}\right)$ for $2 \mathrm{~h}$ subsequent to heat shock, after which they were considered dead if they showed no movement when gently prodded with a blunt probe.

Adult desiccation resistance was measured by placing one- to five-day-old adults maintained at $21 \pm 1^{\circ} \mathrm{C}$ in $2.5 \times 7.5 \mathrm{~cm}$ cylindrical mesh cages in a desiccator maintained at $26.5 \pm 1^{\circ} \mathrm{C}$ for $4.5 \mathrm{~h}$. The 53-L desiccator contained $2.7 \mathrm{~kg}$ of 'Drierite' prepared by heating at $225^{\circ} \mathrm{C}$ for $2 \mathrm{~h}$ and then cooled to $26.5^{\circ} \mathrm{C}$ before using in experiments. Control and selected populations were paired, and each population was run in two blocks on separate days, with 30 control population adults and 30 selected population adults in each block. All blocks were run at $2 \mathrm{PM} \pm 1 \mathrm{~h}$ each day. Adult cages were removed from the desiccator, squirted with water, and survivorship was assessed as described above after two hours.

\section{Statistical analyses}

We used the SAS procedure GLM with type III sums of squares (SAS Institute, 1985) to address several questions relating to the effects of the stressful environment, and the persistence, adaptation and shock tolerance of populations in this experiment. A posteriori pairwise comparison of treatment means was performed using Ryan's $Q$ to control for experiment-wide significance (Day \& Quinn, 1989).

Effects of stress To examine the effects of the stressful environment on the fitness of populations we compared fitness under near-optimal and stressful conditions using measurements we made before initiating selection. We performed ANOVA of $r_{\mathrm{c}}$ using environment (stressful, near-optimal; d.f. $=1$ ) and hybridity (parents, 2-way, 4-way; d.f. $=2$ ) as treatments and populations nested within environment and hybridity as the error term $($ d.f. $=22)$.

Persistence To determine whether fitness $\left(r_{\mathrm{c}}\right)$ under thermally stressful conditions prior to selection predicted persistence, we performed an ANOVA of $r_{\mathrm{c}}$ before selection using persistence/extinction (d.f. $=1)$ and hybridity (parents, 2-way, 4-way; d.f. $=2$ ) as treatments and populations nested within treatments $($ d.f. $=8)$ as the error estimate.

Adaptation We used a single ANOva to test concurrently for (i) adaptation in the selected populations to the stressful environment, and (ii) adaptation in control populations to the laboratory environment in the absence of selection. We performed 2-way ANOvA of $r_{\mathrm{c}}$ in the selected populations that persisted through 10 generations of selection, in their respective control populations, and in the same populations before selection. We used generation (selected, control, before selection; d.f. =2) and hybridity (parents, 2-way, 
4-way; d.f. =2) as treatments and populations nested within treatments $($ d.f. $=12)$ for the error term. This analysis tests for adaptation in the selected lines by simultaneously examining the performance $\left(r_{\mathrm{c}}\right)$ of the selected populations relative to their performance before selection was imposed, and relative to contemporaneous performance of the unselected control populations. This analysis also provides a test for laboratory adaptation, by comparing the performance of the control populations before and after four generations of maintenance in the laboratory as diapausing larvae.

Heat shock All survivorship percentages were arcsinesquare-root transformed to approximate a normal distribution. Preliminary analysis of variance indicated no significant block effect in any of the shock-tolerance trials. Because these tests examine the correlated response of replicate selected populations, populations within selection and hybridity treatments represent the appropriate error term. Therefore, to examine the effects of selection and hybridity on tolerance to transient, acute stress, we performed 2-way ANOVA of survivorship after heat or desiccation shock using selection (selected, control; d.f. =1) and hybridity (parents, 2-way, 4-way; d.f. $=2)$ as treatments with populations nested within selection and hybridity (d.f. $=6$ ) for the error term. An insufficient number of adults was available to measure adult desiccation tolerance in the AL 4-way population. Consequently, for the ANOVA of desiccation tolerance, the remaining ME 4-way population was pooled with the 2-way hybrids, leaving two levels for the hybridity treatment (parental, hybrid; d.f. $=1$ ).

\section{Results}

\section{Persistence}

The stressful environment caused a $54 \%$ reduction in fitness averaged across all populations relative to the near-optimal environment (Fig. 3: $\quad F_{1,22}=291.32$; $P<0.001)$. Hybridity affected fitness $\left(F_{2,22}=5.97\right.$; $P=0.023)$ but environment-by-hybridity interaction did not $\left(F_{2,22}=1.49 ; P=0.247\right)$. A posteriori comparison of treatment means indicated that 2-way hybrid populations had significantly higher fitness than parental and 4-way hybrid populations, which did not differ significantly (Fig. 3, inset). These results indicate that the stressful environment we utilized did impose a

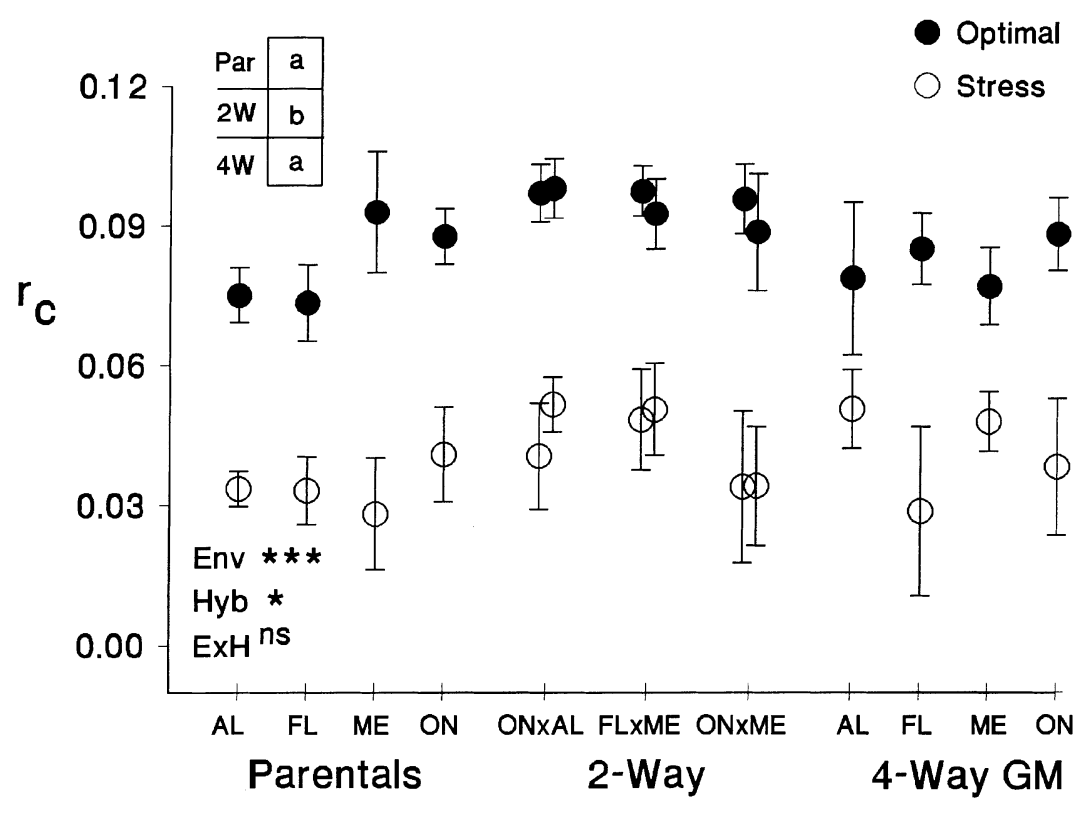

Fig. 3 Capacity for increase $\left(r_{c} \pm 2 \mathrm{SE}\right)$ of parental, 2-way hybrid (2-Way), and 4-way hybrid (4-Way GM) populations of Wyeomyia smithii under optimal (solid circles) and stressful (open circles) conditions prior to the initiation of selection (before selection). Reciprocal 2-way hybrid populations are positioned adjacently with respect to maternal ( + ) contribution by left/right

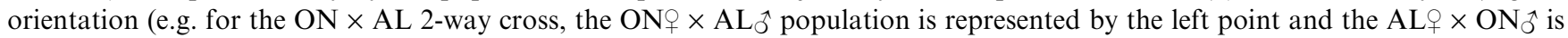
represented by the right point). The 4-way hybrid populations are labelled according to grandmaternal (GM) contribution. The legend in the lower left indicates results of ANOva testing for effects of environment (Env), hybridity (Hyb), and environmentby-hybridity interaction $(\mathrm{E} \times \mathrm{H})$. ${ }^{\text {ns }}$ indicates $P>0.05,{ }^{*} P<0.05, * * * P<0.001$. The legend in the upper left indicates results of Ryan's $Q$-test in which treatment means associated with the same letter $(\mathrm{a}, \mathrm{b})$ are not significantly different $(P>0.05)$; Par, Parental; 2W, 2-way hybrid; 4W, 4-way hybrid. 
substantial cost to fitness, and that 2-way hybrid populations, but not 4-way hybrid populations, displayed hybrid vigour (heterosis).

During 10 generations of selection in the stressful environment, exactly one-half of the parental, one-half of the 2-way, and one-half of the 4-way hybrid populations persisted and the others went extinct (Fig. 4). Thus hybrid populations were no more likely to persist than parental populations.

We were able to compare retrospectively the fitness in each of the 14 populations before selection according to whether that population eventually persisted or went extinct (Fig. 4). Fitness $\left(r_{\mathrm{c}}\right)$ before selection under stressful conditions was higher in populations that eventually persisted than in populations that eventually went extinct $\left(F_{1,8}=14.02 ; P=0.006\right)$, differed significantly because of hybridity $\left(F_{2,8}=6.63 ; P=0.020\right)$, and was affected by a significant persistence-by-hybridity interaction $\left(F_{2,8}=9.69 ; P=0.007\right)$. Ryan's $Q$ pairwise comparison (Fig. 4, inset) showed that 2-way and 4-way hybrid populations that persisted did not differ in fitness from each other, but that these populations had significantly higher fitness than parental populations that persisted and 2-way and 4-way populations that went extinct. Fitness of the parental populations before selection did not differ between parental populations that ultimately went extinct and those that ultimately persisted (Fig. 4, inset). These results show that fitness in the parental populations before selection did not predict their persistence. However, fitness in the hybrid populations before selection was higher in populations destined to persist than in populations destined to go extinct, regardless of the degree of hybridity.

\section{Adaptation}

For populations that persisted through 10 generations of selection (Fig. 5), neither generation (selected, control, before selection; $F_{2,12}=0.30 ; P=0.746$ ) nor hybridity (parental, 2-way, 4-way; $F_{2,12}=0.06 ; P=0.942$ ) affected fitness under stressful conditions. Thus, we found no evidence for adaptation of the selected populations to the stressful environment, and no evidence for changes in fitness of the control populations. Subsequent analyses also indicated no effect of selection on the component traits of $r_{\mathrm{c}}$ (fertility, survivorship, fecundity, generation time), and in the interests of brevity the details of these analyses have been omitted.

However, a generation-by-hybridity interaction did affect fitness $\left(F_{4,12}=9.81 ; P<0.001\right)$. A posteriori comparison of means (Fig. 5, inset) showed that this significant interaction was caused by a lower fitness of the

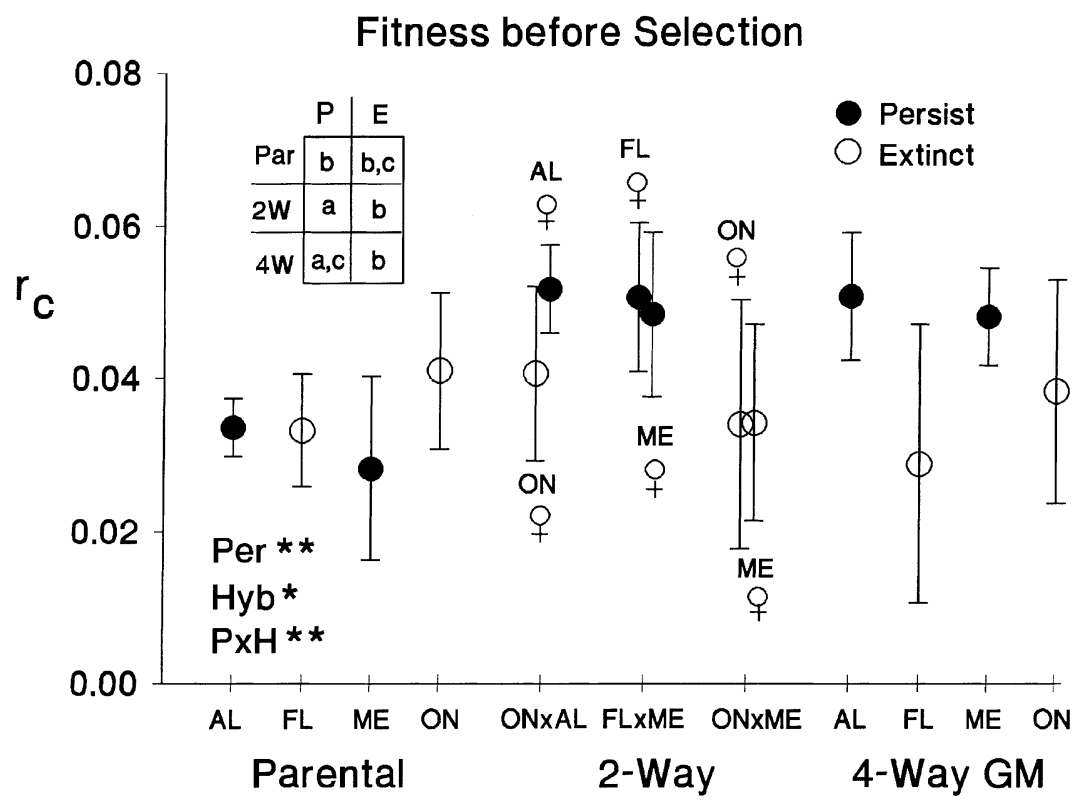

Fig. 4 Capacity for increase $\left(r_{\mathrm{c}} \pm 2 \mathrm{SE}\right)$ of parental, 2-way hybrid (2-Way), and 4-way hybrid (4-Way GM) populations of Wyeomyia smithii under stressful conditions prior to the initiation of selection (before selection). Reciprocal 2-way hybrid populations are positioned adjacently and labelled by maternal (†) contribution. The 4-way hybrid populations are labelled as in Fig. 3. Open circles indicate populations which went extinct during 10 generations of selection, closed circles indicate populations which persisted. The legend in the lower left indicates results of ANOva testing for effects of persistence (Per), hybridity (Hyb), and persistence-by-hybridity interaction $(\mathrm{P} \times \mathrm{H})$. Significance is denoted as in Fig. 3, except $* * P<0.01$. The legend in the upper left indicates results of Ryan's $Q$-test as in Fig. 3, but with $\mathrm{P}=$ persistent, $\mathrm{E}=$ extinct.

(C) The Genetical Society of Great Britain, Heredity, 83, 509-519. 


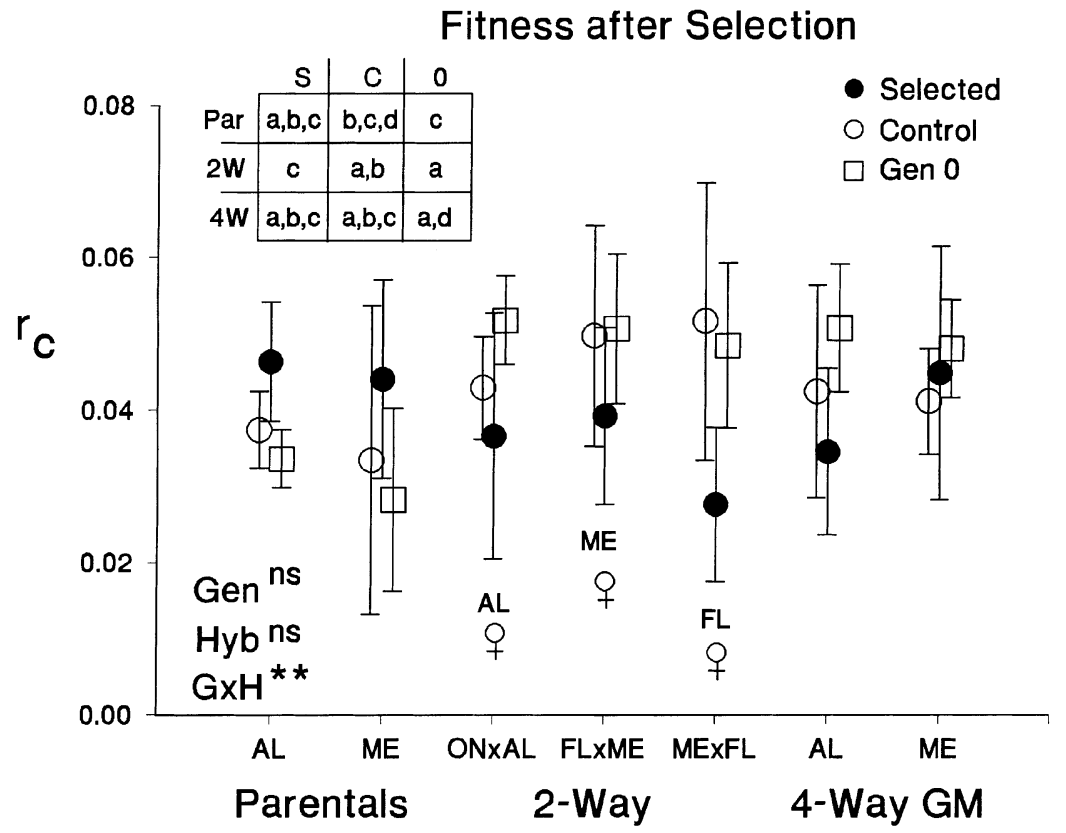

Fig. 5 Capacity for increase $\left(r_{\mathrm{c}} \pm 2 \mathrm{SE}\right)$ measured before selection (Gen. $0=$ before selection, open squares), and in control (open circles) and selected (solid circles) populations of Wyeomyia smithii that persisted through 10 generations of selection. Population labels as in Fig. 4. The legend in the lower left shows the results of ANOva testing for effects of generation (Gen), hybridity ( $\mathrm{Hyb})$, and generation by hybridity interaction $(G \times H)$. Significance as in Figs 3 and 4 . The legend in the upper left indicates results of Ryan's $Q$-test as in Fig. 3, but with $\mathrm{S}$, selected; C, control; 0 , Gen. 0 , before selection. 2-way hybrid selected populations relative to both the 2-way hybrid control and the 2-way hybrid populations before selection. These results show that selection in the chronically stressful environment did not lead to improved fitness in that environment and, in fact, resulted in reduced fitness in the 2-way hybrid populations.

\section{Shock tolerance}

Survivorship through larval heat shock (Fig. 6a) was higher in the selected than in the control populations $\left(F_{1,6}=7.30 ; P=0.035\right)$ but neither hybridity $\left(F_{2,6}=2.61\right.$; $P=0.153)$ nor selection-by-hybridity interaction $\left(F_{2,6}=0.15 ; P=0.864\right)$ affected survivorship. Survivorship through adult heat shock (Fig. 6b) was higher in the selected than in the control populations $\left(F_{1,6}=12.05 ; P=0.013\right)$ and was affected by hybridity $\left(F_{2,6}=25.21 ; \quad P=0.001\right)$ but not by a selectionby-hybridity interaction $\left(F_{2,6}=1.13 ; P=0.383\right)$. Ryan's $Q$ pairwise comparisons (Fig. 6b, inset) showed that hybrid populations, regardless of the level of hybridity, survived better than parental populations. Survivorship through adult desiccation stress was higher in the selected than in the control populations $\left(F_{1,4}=40.01\right.$; $P=0.003)$ but hybridity did not affect survivorship $\left(F_{1,4}=0.15 ; P=0.718\right)$ nor did a selection-by-hybridity interaction $\left(F_{1,4}=0.39 ; P=0.566\right)$.

\section{Discussion}

The conditions of temperature, food level and humidity in the stressful environment of this study were specifi- cally designed to impose broad physiological stress over the whole life-cycle of $W$. smithii. Comparison of fitness under these stressful conditions relative to fitness in a near-optimal environment (Fig. 3) shows that the stressful environment we utilized led to a $54 \%$ decrease in fitness averaged across all populations. Thus, the stressful environment did impose a cost to fitness consistent with classical definitions of 'stress' as a factor causing potentially injurious change to a biological system (Hoffmann \& Parsons, 1991).

As planned at the initiation of these experiments, half of the initial populations went extinct during the course of 10 generations of selection under stressful conditions. Exactly one-half of the parental, 2-way, and 4-way hybrid populations went extinct (Fig. 4). Thus, hybridization per se did not enhance the likelihood of population persistence over 10 generations.

Among the hybrid populations, fitness before selection was higher in hybrid populations that persisted than in hybrid populations that ultimately went extinct (Fig. 4). However, fitness did not differ between parental populations that ultimately went extinct and parental populations that ultimately persisted during 10 generations of selection. Thus a comparison of fitness among the hybrid populations before selection was initiated was more likely to be indicative of persistence under stressful conditions than a comparison of fitness among parental populations.

In the seven populations persisting through 10 generations of selection, neither selection nor hybridity affected fitness $\left(r_{\mathrm{c}}\right)$ under stressful conditions (Fig. 5). However, a significant selection-by-hybridity interaction 

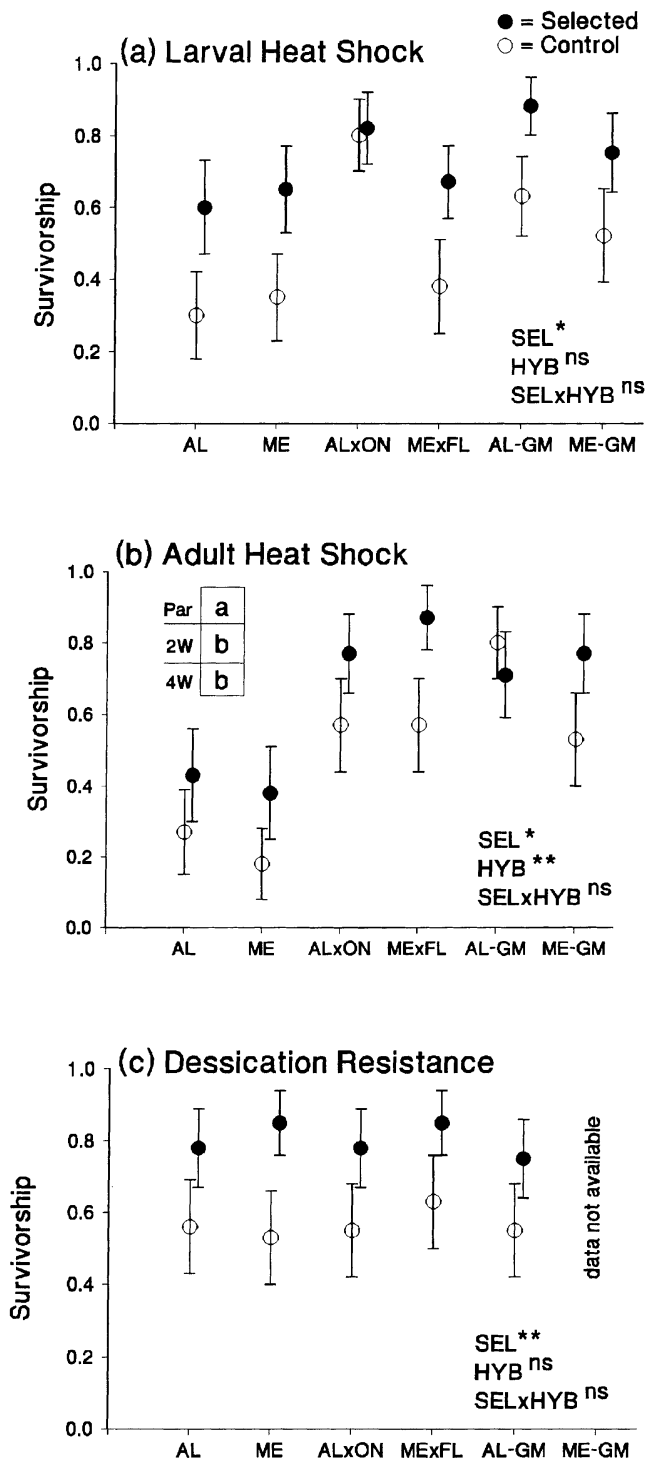

Fig. 6 Survivorship of (a) larval heat shock (b) adult heat shock, and (c) desiccation shock, in six of seven populations of Wyeomyia smithii that persisted through 10 generations of selection. Symbols and conventions as in Figs 3 and 4 except that 2-way hybrid populations are labelled by the specific cross. An insufficient number of individuals was available to test desiccation resistance in the ME-GM population (see text).

did affect fitness, and subsequent pairwise comparisons showed a decrease, not increase, in performance of the 2-way hybrid selected populations relative to their control populations and fitness before selection (Fig. 5, inset). Thus, hybridization in the 2-way, but not the 4-way populations, constrained rather than facilitated adaptation to stressful conditions.

The results of this experiment indicate that hybridization did not increase either the likelihood of population persistence under, or adaptation to, a chronically stressful environment, despite initially beneficial effects of hybridization to fitness (Figs 3 and 4). We therefore suggest that attempts to augment genetic variation by outcrossing differentiated populations should be undertaken cautiously (Spielman \& Frankham, 1992). As in this experiment, initially beneficial effects of hybridization may be transient, and may be balanced or outweighed by deleterious effects which are only apparent in the longer term. The latent deleterious effects of hybridization, an outbreeding depression not apparent until generations subsequent to the $F_{1}$ and $F_{2}$, may be caused by recombination in generations subsequent to the $F_{2}$ breaking apart favourable epistatic gene systems. Extensive work has demonstrated the importance of such gene systems to the underlying genetic architecture of both demographic and phenological traits in W. smithii (Hard et al., 1993; Armbruster et al., 1997; Lair et al., 1997).

Larval and adult heat-shock tolerance and adult desiccation resistance all increased in response to our selection regime. These traits therefore appear to be part of a generalized shock-resistance phenotype. In D. melanogaster, resistance to starvation, desiccation, and toxic concentrations of ethanol have been shown to evolve in concert (Service et al., 1985; but see Graves et al., 1992), as have resistance to heat shock, desiccation, Cobalt- $\delta$-radiation, and acute levels of ethanol and acetic acid (Hoffmann \& Parsons, 1989).

Although many comparative studies demonstrate a robust relationship between thermal tolerance and prevailing temperature at the locality of origin (Hoffmann \& Parsons, 1991; Krebs \& Loeschcke, 1995), only four studies of which we are aware, all utilizing D. melanogaster (Stephanou \& Alahiotis, 1983; Huey et al., 1991; Cavicchi et al., 1995; Gilchrist et al., 1997), have examined the joint evolution of thermal adaptation and shock tolerance within laboratory populations. Although the experimental design employed in these Drosophila studies does not provide a definitive test of thermal adaptation (Partridge et al., 1995), all of these studies found a general, positive correlation between the thermal regime under which population cages were maintained for 60 or more generations and subsequent heat-shock tolerance. In contrast to these results, our data indicate that, within populations of W. smithii, heat-shock tolerance and fitness $\left(r_{\mathrm{c}}\right)$ evolved independently and that shock tolerance was adaptively more labile than fitness. It is possible that the strong response of shock-tolerance traits in the selected populations, which occurred despite no improvement in overall fitness, represents an initial or cryptic response to selection for improved fitness under environmentally stressful conditions. These results provide the caveat that simple extrapolation from shock tolerance to 
fitness in stressful environments may be misleading. In evaluating how organisms might respond to future climatic change, a clear distinction must be maintained between survivorship through transient, acute stress and performance of fitness traits through life-long chronic stress.

\section{Acknowledgements}

We thank R. Lande and M. Lynch for suggestions relating to experimental design, and $\mathrm{C}$. Kleckner and $\mathrm{K}$. Lair for assistance in running experiments. A.F. Bennet, R.B. Huey, and two anonymous reviewers provided helpful comments on earlier versions of this manuscript. This work was supported by NSF grant DEB-9305584 to W.E.B, NSF training grant BIR-9014265 and a University of Oregon Doctoral Research Fellowship to P.A.

\section{References}

ANDREWARTHA, H. G. AND BIRCH, L. C. 1954. The Distribution and Abundance of Animals. University of Chicago Press, Chicago, IL.

ARMBRUSTER, P., BRADSHAW, W. E. AND HOLZAPFEL, C. M. 1997. Evolution of the genetic architecture underlying fitness in the pitcher-plant mosquito, Wyeomyia smithii. Evolution, 51, 451-458.

BARTlein, P. J. AND PRENTICE, I. C. 1989. Orbital variations, climate, and paleoecology. Trends Ecol. Evol., 4, 195-199.

BRADSHAw, w. E. 1980. Thermoperiodism and the thermal environment of the pitcher-plant mosquito, Wyeomyia smithii. Oecologia, 46, 13-17.

BRADSHAW, w. E. 1983. Interaction between the mosquito Wyeomyia smithii, the midge, Metriocnemus knabi, and their carnivorous host Sarracenia purpurea. In: Frank, J. H. and Lounibos, L. P. (eds) Phytotelmata: Terrestrial Plants as Hosts for Aquatic Insect Communities, pp. 161-189. Plexus Publishing, Medford, NJ.

BRADSHAw, w. E. AND HOLZAPFEL, C. M. 1989. Life-historical consequences of density-dependent selection in the pitcherplant mosquito, Wyeomyia smithii. Am. Nat., 133, 869-887.

BRADSHAW, W. E. AND HOLZAPFEL, C. M. 1992. Reproductive consequences of density-dependent size variation in the pitcher-plant mosquito, Wyeomyia smithii (Diptera: Culicidae). Ann. Entomol. Soc. Am., 85, 274-281.

BRADSHAW, W. E. AND LOUNIBOS, L. P. 1977. Evolution of dormancy and its photoperiodic control in pitcher-plant mosquitoes. Evolution, 31, 546-567.

CAVICCHI, S., GUERRA, D. L. A., TORRE, V. AND HUEY, R. B. 1995. Chromosomal analysis of heat-shock tolerance in Drosophila melanogaster evolving at different temperatures in the laboratory. Evolution, 49, 676-684.

CRILL, W. D., HUEY, R. B. AND GILCHRIST, G. W. 1996. Within- and between-generation effects of temperature on the morphology and physiology of Drosophila melanogaster. Evolution, 50, 1205-1218.

DAY, R. W. AND QUINN, G. P. 1989. Comparisons of treatments after analysis of variance in ecology. Ecol. Monogr., 59, 433-463.

GILCHRIST, G. W., HUEY, R. B. AND PARTRIDGE, L. 1997. Thermal sensitivity of Drosophila melanogaster: Evolutionary responses of adults and eggs to laboratory natural selection at different temperatures. Physiol. Zool., 70, 403-414.

GRAVES, J. L., TOOLSON, E. C., JEONG, C., VU, L. N. AND ROSE, M. R. 1992. Desiccation, flight, glycogen, and postponed senescence in Drosophila melanogaster. Physiol. Zool., 65, 268-286.

HARD, J. J., BRADSHAw, W. E. AND HOLZAPFEL, C. M. 1993. The genetic basis of photoperiodism and evolutionary divergence among populations of the pitcher-plant mosquito, Wyeomyia smithii. Am. Nat., 142, 457-473.

HOFFMANN, A. A. AND PARSONS, P. A. 1989. Selection for increased desiccation resistance in Drosophila melanogaster: additive genetic control and correlated responses to other stresses. Genetics, 122, 837-845.

HOFFMANN, A. A. AND PARSONS, P. A. 1991. Evolutionary Genetics and Environmental Stress. Oxford University Press, New York.

HUEY, R. B., PARTRIDGe, L. AND FOWLER, K. 1991. Thermal sensitivity of Drosophila melanogaster responds rapidly to laboratory natural selection. Evolution, 45, 751-756.

KAREIVA, P. M., KINGSOlVER, J. G. AND HUEY, R. B. (eds) 1993. Biotic Interactions and Global Change. Sinauer Associates, Sunderland, MA.

KATTENBERG, A. F., GIORGI, F., GRASSL, H., MEEHL, G. A., MITCHELL, J. F. B. AND STOUGGER, R. J. ET $A L$. 1996. Climate models - Projections of future climate. In: Houghton, J. T., Meira Filho, L. G., Chandler, B. A., Harris, N., Kattenberg, A. F. and Maskel, K. (eds) Climate Change 1995. The Science of Climate Change. Contribution I to the Second Assessment Report of the Intergovernmental Panel on Climate Change, pp. 285-357. Cambridge University Press, Cambridge.

KREBS, R. A. AND LOESCHCKE, V. 1995. Resistance to thermal stress in preadult Drosophila buzzati: variation among populations and changes in relative resistance across life stages. Biol. J. Linn. Soc., 56, 517-531.

LAIR, K. P., BRADSHAw, W. E. AND HOLZAPFEL, C. M. 1997. Evolutionary divergence of the genetic architecture underlying photoperiodism in the pitcher-plant mosquito, Wyeomyia smithii. Genetics, 147, 1873-1883.

LYNCH, M. 1991. The genetic interpretation of inbreeding depression and outbreeding depression. Evolution, 45, 622-629.

LYNCH, M. AND LANDE, R. 1993. Evolution and extinction in response to environmental change. In: Kareiva, P. M., Kingsolver, J. G. and Huey, R. B. (eds) Biotic Interactions and Global Climate Change, pp. 234-250. Sinauer Associates, Sunderland, MA.

MOEUR, J. E. AND ISTOCK, C. A. 1980. Ecology and evolution of the pitcher-plant mosquito IV. Larval influence over adult reproductive performance and longevity. J. Anim. Ecol., 49, 775-792. 
PARTRIDGE, L., BARRIE，B., BARTON, N. H., FOWLER, K. AND FRENCH, V. 1995. Rapid laboratory evolution of adult lifehistory traits in Drosophila melanogaster in response to temperature. Evolution, 49, 538-544.

PITTENDRIGH, C. S. 1961. On temporal organization in living systems. Harvey Lectures, 56, 93-125.

SAS INSTITUTE. 1985. SAS User's Guide: Statistics, version 5. SAS Institute Inc., Cary, NC.

SERVICE, P. M., HUTCHINSON, E. W., MACKINLEY, M. D. AND ROSE, M. R. 1985. Resistance to environmental stress in Drosophila melanogaster selected for postponed senescence. Physiol. Zool., 58, 380-389.

SPIELMAN, D. AND FRANKHAM, R. 1992. Modeling problems in conservation genetics using captive Drosophila populations: Improvement of reproductive fitness due to immigration of one individual into small partially inbred populations. Zool. Biol., 11, 343-351.

STEPHANOU, G. AND ALAhiotis, S. N. 1983. Non-Mendelian inheritance of 'heat-sensitivity' in Drosophila melanogaster. Genetics, 103, 93-107. 